\title{
Constraining the Origin of Local Positrons with HAWC TeV Gamma-Ray Observations of Two Nearby Pulsar Wind Nebulae
}

\author{
R. López-Coto ${ }^{* a}$, S. Casanova ${ }^{a, b}$, B. Dingus ${ }^{c}$, F. Salesa-Greus ${ }^{b}$ and H. Zhou ${ }^{c}$ \\ ${ }^{a}$ Max-Planck-Institut für Kernphysik \\ Saupfercheckweg 1, Heidelberg, BW 69117, Germany \\ ${ }^{b}$ Instytut Fizyki Jadrowej im. Henryka Niewodniczańskiego, Polskiej Akademii Nauk \\ ul. Radzikowskiego 152, 31-342 Krakow, Poland \\ ${ }^{c}$ Physics Division, Los Alamos National Laboratory \\ Los Alamos, NM 87545, USA \\ E-mail: rlopez@mpi-hd.mpg.de, sabrina.casanova@ifj.edu.pl, \\ dingus@lanl.gov, fsalesa@ifj.edu.pl, hzhoulemtu.edu
}

\section{for the HAWC Collaboration ${ }^{\dagger}$}

\begin{abstract}
Cosmic-ray positrons are charged antiparticles that strike Earth's atmosphere isotropically. At energies below several $\mathrm{GeV}$ they are produced by cosmic-ray protons interacting with nearby interstellar matter. At higher energies, an unexpected and unexplained excess above the protoninduced background has been detected by several satellites, including PAMELA, AMS-02 and Fermi. Due to energy losses in interstellar magnetic and radiation fields, the highest-energy positrons observed must have originated in our immediate Galactic neighborhood. This excess has been theorized to be originated from nearby astrophysical sources, dark matter, or new modes of cosmic-ray secondary production. Amongst the astrophysical sources, pulsars as Geminga and PSR B0656+14 have been proposed to be contributors to this excess. The HAWC GammaRay Observatory reported the discovery of $\mathrm{TeV}$ gamma-ray emission extending several degrees around the positions of Geminga and PSR B0656+14 pulsars. Using the morphological and spectral measurements of these two VHE gamma-ray sources, we determine the diffusion coefficient of electrons escaping them and their contribution to the positron flux measured at Earth. For this assumption of isotropic diffusion, we find that neither of these sources make an appreciable contribution to the locally measured positron flux.
\end{abstract}

7th Fermi Symposium 2017

15-20 October 2017

Garmisch-Partenkirchen, Germany

\footnotetext{
* Speaker.

${ }^{\dagger}$ Complete list of authors at http://www.hawc-observatory.org/collaboration/icrc2017.php
} 


\section{Introduction}

Cosmic rays (CRs) are high-energy charged particles that strike the atmosphere almost isotropically. They are composed by protons and helium nuclei (99\%), heavier nuclei, electrons $\left(\mathrm{e}^{-}\right)$, positrons $\left(\mathrm{e}^{+}\right)$, antiprotons and neutrinos. There are galactic propagation models that reproduce the all-electron $\left(\mathrm{e}^{ \pm}\right) \mathrm{CR}$ energy. However, the $\mathrm{e}^{+}$content in the total $\mathrm{e}^{ \pm}$flux above $10 \mathrm{GeV}$ (also known as "positron excess") measured by PAMELA and AMS amongst others, can only be explained if there is a source injecting them. Moreover, taking into account standard diffusion and cooling of $\mathrm{e}^{ \pm}$, the extension of the $\mathrm{e}^{ \pm}$spectrum up to $\mathrm{TeV}$ energies can only be explained if the source is nearby ( $\sim$ hundreds of parsecs) and middle age ( $\sim$ hundred of kyr). Pulsars, as factories of $\mathrm{e}^{ \pm}$, are the most suitable candidates to explain the origin of this excess. Amongst the known pulsars, Geminga and PSR J0659+14 are the two best candidates due to their distance and age. Since $\mathrm{e}^{ \pm}$are charged particles, their arrival direction does not point to their origin because they are deflected by magnetic fields. One of the ways to study sources of CRs is to analyse the neutral subproducts of CR collisions such as gamma rays. Establish that pulsars are not the origin of this excess would point to more exotic processes such as galactic jets [1] or dark matter [2]. Milagro reported very-high-energy (VHE; E >100 GeV) gamma-ray emission from Geminga in 2009 [3]. The emission was very extended $\left(2.6^{\circ}\right)$ and had a significance of $6.3 \sigma$. The Tibet Air shower array reported an excess of $2.2 \sigma$ at the location of the pulsar but did not report extended emission [4]. Imaging Atmospheric Cherenkov Telescopes (IACTs) have observed Geminga without significant detection $[5,6]$.

\section{HAWC Observatory and dataset}

The HAWC Gamma-Ray Observatory is located at Sierra Negra, Mexico at $4100 \mathrm{~m}$ a.s.l., and is sensitive to gamma rays and cosmic rays in the energy range from $100 \mathrm{GeV}$ to $100 \mathrm{TeV}$ [7]. It is composed by 300 optically isolated tanks covering an area of $22000 \mathrm{~m}^{2}$. Each one of these Water Cherenkov Detectors (WCD) consists of a metallic cylinder of $7.3 \mathrm{~m}$ diameter and $4.5 \mathrm{~m}$ height containing 180000 liters of water. They are equipped with one 10" PMT at the center and three 8" PMTs surrounding the central one. The array has a 2 sr field of view with $>95 \%$ uptime. It started operation in its full configuration in March 2015. The current analysis was performed using 17 months of data with the complete HAWC array, the same dataset used for the HAWC $2 \mathrm{HWC}$ catalog [8]. In the catalog, the detection of extended emission in the vicinity of Geminga and PSR B0656+14 was reported associated with the HAWC source 2HWC J0635+180 and 2HWC J0700+143.

\section{Results}

The Geminga pulsar (PSR J0633+1746) is an isolated neutron star in the Gemini constellation. The characteristic age of the pulsar is $3.42 \times 10^{5} \mathrm{yr}$ and the distance of $250 \mathrm{pc}$ with a spin-down luminosity of $3.26 \times 10^{34} \mathrm{erg} \mathrm{s}^{-1}$ [9]. The pulsar B0656+14 is also an isolated neutron star which has been associated with the large ( 25 degree) supernova remnant covering the Gemini and Monoceros constellations called the "Monogem ring". Some authors speculated that the Monogem Ring SNR 
could be the long-searched-for source of the highest energy protons producing the "knee" feature in the cosmic-ray spectrum [10]. PSR B0656+14 has a characteristic age is $1.1 \times 10^{5} \mathrm{yr}$, the distance to Earth is $288 \mathrm{pc}$, and the spin-down luminosity is $3.8 \times 10^{34} \mathrm{erg} \mathrm{s}^{-1}$ [9].

For the analysis, we tested different morphological assumptions as shown in Fig. 2. The first morphology approximated the source as extended disks where a constant (average) flux of gamma-rays fitted within a distance from the source center location. The second morphology is a symmetrical two-dimensional Gaussian. In this case, the fitted flux of gamma-rays depends on the distance from the source center location as following a 2D-Gaussian. Apart from these two morphologies, which do not depend on physical assumptions, a third one based on [11] assumes electron and positron pairs diffusing into the interstellar medium around the pulsar, producing gamma rays through inverse Compton scattering of CMB photons.

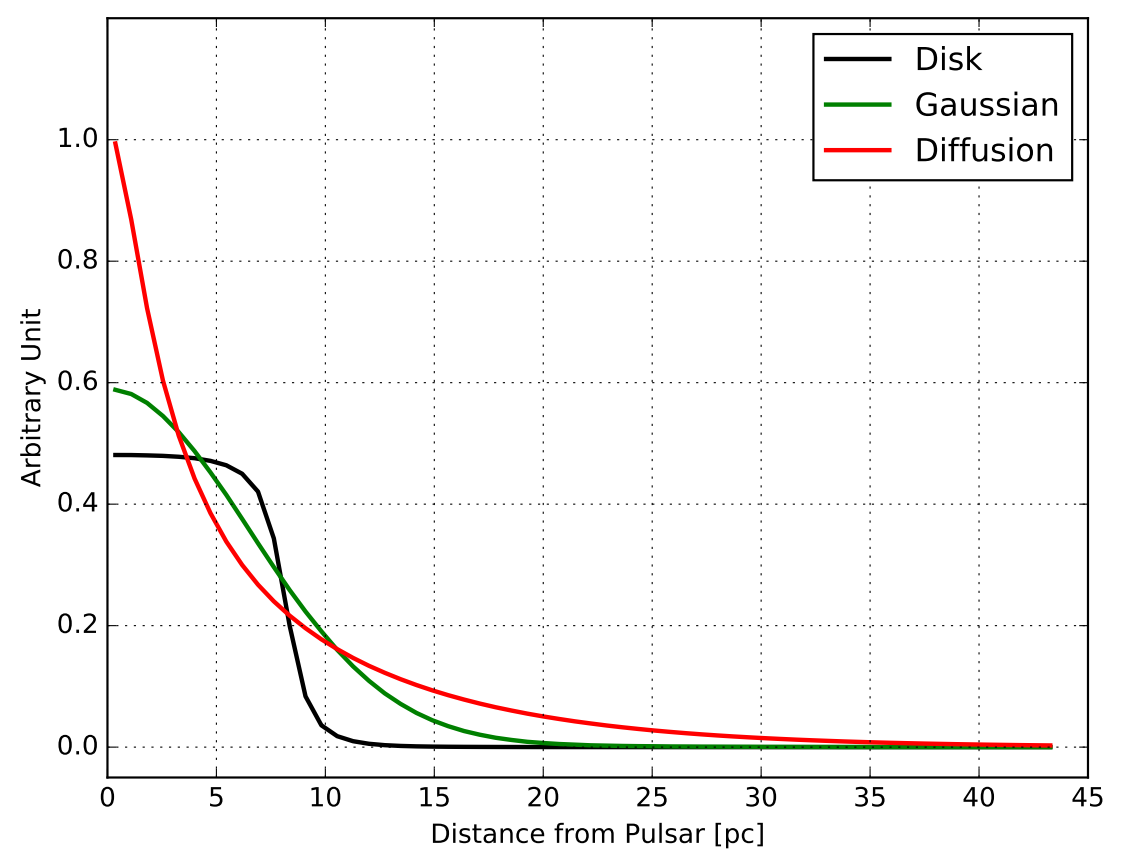

Figure 1: Geminga flux per surface unit for different radial distances. Overlaid are the three morphological models tested.

The results show that the emission from the Geminga location reaches $13.1 \sigma$ when a diffusion model morphology is used (see Fig. 1). In addition, a similar extended TeV gamma-ray emission in the vicinity of PSR B0656+14 has been detected at an $8.1 \sigma$ level. Unlike Geminga, the extended emission of PSR B0656+14 was not reported before by any gamma-ray experiment.

A first estimate of the source gamma-ray flux was provided in the recent HAWC catalog [8]. The final result using a diffusion model morphology can be found in [12].

\section{Conclusion}

HAWC carried out morphological and spectral studies using the 17 moths dataset of the Geminga and PSR B0656+14 region. These studies provide information about the diffusion co- 

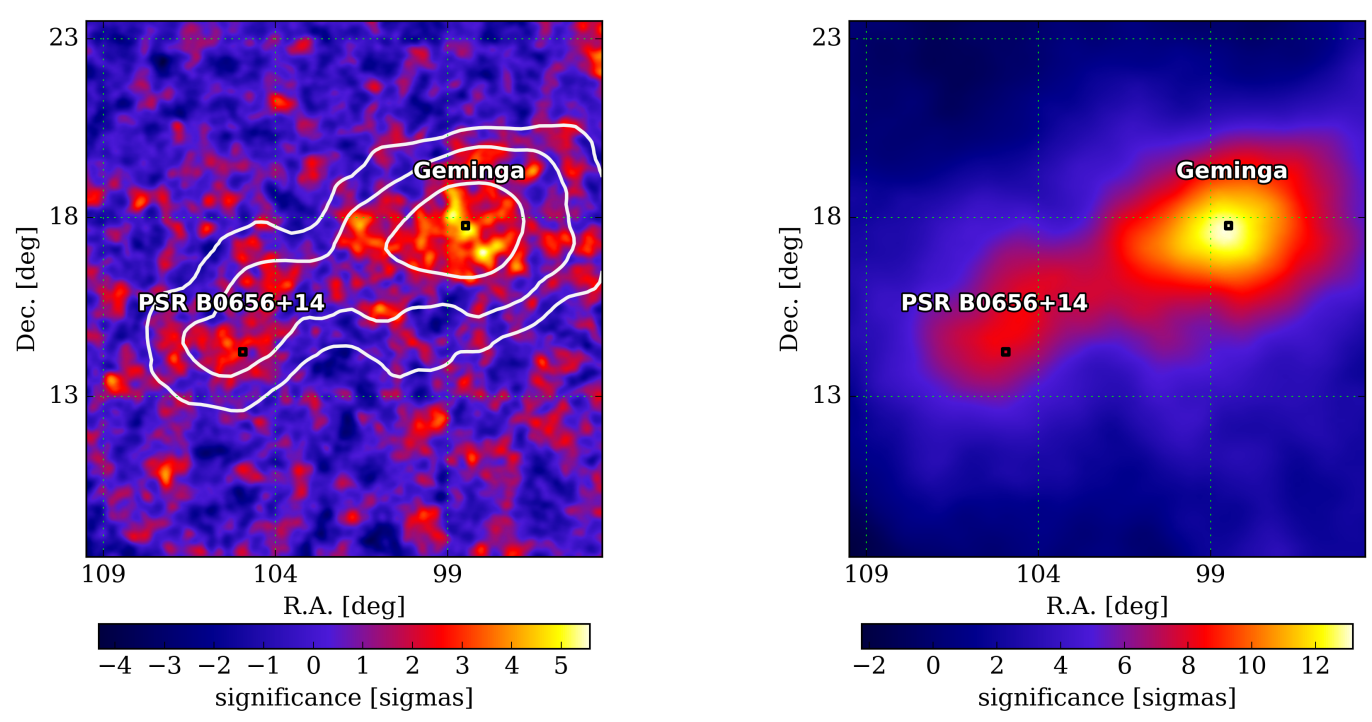

Figure 2: Significance map for the Geminga and PSR B0656+14 region convolved with the point spread function (left) and with the particle diffusion morphology (right).

efficient and gamma-ray spectrum which can be used as input parameters for the EDGE code [13]. EDGE takes into account the theory of electron diffusion, and computes the expected positron flux produced at the Earth by a pulsar. The derived positron spectrum at Earth considering the measurements of Geminga and PSR B0656+14 by HAWC and further conclusions are shown in [12]

\section{Acknowledgments}

We acknowledge the support from: the US National Science Foundation (NSF); the US Department of Energy Office of High-Energy Physics; the Laboratory Directed Research and Development (LDRD) program of Los Alamos National Laboratory; Consejo Nacional de Ciencia y Tecnología (CONACyT), México (grants 271051, 232656, 260378, 179588, 239762, 254964, 271737, 258865, 243290, 132197), Laboratorio Nacional HAWC de rayos gamma; L'OREAL Fellowship for Women in Science 2014; Red HAWC, México; DGAPA-UNAM (grants IG100317, IN111315, IN111716-3, IA102715, 109916, IA102917); VIEP-BUAP; PIFI 2012, 2013, PROFOCIE 2014, 2015; the University of Wisconsin Alumni Research Foundation; the Institute of Geophysics, Planetary Physics, and Signatures at Los Alamos National Laboratory; Polish Science Centre grant DEC-2014/13/B/ST9/945; Coordinación de la Investigación Científica de la Universidad Michoacana. Thanks to Luciano Díaz and Eduardo Murrieta for technical support.

\section{References}

[1] Gupta, N. et al., $p \gamma$ interactions in Galactic jets as a plausible origin of the positron excess, Mon. Not. Roy. Astron. Soc. 441 (4) (2014) 3122-3126.

[2] Ibarra, A., et al., Dark matter annihilations and decays after the AMS-02 positron measurements, Phys. Rev. D89 (6) (2014) 063539.

[3] Abdo, A. A. et al., Milagro Observations of TeV Emission from Galactic Sources in the Fermi Bright Source List, Astrophys. J. 700 (2009) L127-L131. 
[4] Amenomori, M., et al., Observation of TeV Gamma Rays from the Fermi Bright Galactic Sources with the Tibet Air Shower Array, ApJ 709 (2010) L6-L10. arXiv: 0912.0386.

[5] Maier, G., Observation of Galactic Gamma-ray Sources with VERITAS, in: Aharonian, F. A., et al. (Eds.), American Institute of Physics Conference Series, Vol. 1085 of American Institute of Physics Conference Series, 2008, pp. 187-190. arXiv:0810.0515.

[6] Ahnen, M. L., et al., Search for VHE gamma-ray emission from Geminga pulsar and nebula with the MAGIC telescopes, A\&A591 (2016) A138. arXiv:1603.00730.

[7] Abeysekara, A. U., et al., Sensitivity of the high altitude water Cherenkov detector to sources of multi-TeV gamma rays, Astroparticle Physics 50 (2013) 26-32. arXiv:1306.5800.

[8] Abeysekara, A. U., et al., The 2HWC HAWC Observatory Gamma-Ray Catalog, ApJ843 (2017) 40. arXiv: 1702.02992 .

[9] Manchester, R. N., et al., The Australia Telescope National Facility Pulsar Catalogue, AJ129 (2005) 1993-2006. arXiv:astro-ph/0412641.

[10] Erlykin, A. D. et al., Cosmic rays and the Monogem supernova remnant, Astroparticle Physics 22 (2004) 47-63. arXiv:astro-ph/0404530.

[11] Aharonian, F. A., et al., High energy electrons and positrons in cosmic rays as an indicator of the existence of a nearby cosmic tevatron, Astron. Astrophys. 294 (1995) L41-L44.

[12] Abeysekara, A. U., et al., Extended TeV Gamma-Ray Sources Around Pulsars Constrain the Origin of the Positron Flux at Earth, Science, in print.

[13] Lopez-Coto, R., et al., EDGE: a code to calculate diffusion of cosmic-ray electrons and their gamma-ray emission, ArXiv e-printsarXiv:1708.03139.

[14] Yüksel, H., et al., TeV Gamma Rays from Geminga and the Origin of the GeV Positron Excess, Phys. Rev. Lett. 103 (2009) 051101.

[15] Baughman, B. M., et al., TeV Gamma-Ray Emission Observed from Geminga with HAWC arXiv:1508. 03497.

[16] Zhou, H. et al., TeV Gamma-Ray Observations of Geminga with HAWC, in: APS Meeting Abstracts, 2016. 